08

\title{
Модельные оценки квантовой емкости графеновых наноструктур
}

\author{
() С.Ю. Давыдов ${ }^{1}$, А.А. Лебедев ${ }^{1}$, П.В. Булат ${ }^{2,3}$, А.В. Зубов ${ }^{2}$ \\ ${ }^{1}$ Физико-технический институт им. А.Ф. Иофффе РАН, Санкт-Петербург, Россия \\ ${ }^{2}$ Университет ИТМО, Санкт-Петербург, Россия \\ ${ }^{3}$ Севастопольский государственный университет, Севастополь, Россия \\ E-mail: Sergei_Davydov@mail.ru
}

Поступило в Редакцию 27 марта 2020 г.

В окончательной редакции 27 марта 2020г.

Принято к публикации 16 апреля 2020г.

\begin{abstract}
В рамках простых моделей получены аналитические оценки квантовой емкости $C_{Q}$ для бесконечного листа графена, графеновой наноленты и цепочки атомов углерода (карбина). Продемонстрирована немонотонная зависимость $C_{Q}$ от электростатического потенциала. Полученные результаты сопоставлены с расчетами других авторов.
\end{abstract}

Ключевые слова: однолистный графен, графеновая нанолента, карбин, плотность состояний.

DOI: 10.21883/PJTF.2020.15.49739.18317

Понятие квантовой емкости $C_{Q}$ было впервые сформулировано для двумерного электронного газа в работе [1]. Среди ранних оценок $C_{Q}$ для графеновых наноструктур отметим работы [2-4]; современное состояние проблемы отражено в обзорах [5-7]. К настоящему времени значения $C_{Q}$ рассчитаны для двумерного электронного газа $[1,2]$, однолистного [3,5-7] и двухлистного [5,7] графенов, эпитаксиального графена на карбиде кремния [8] и графеновой наноленты $[3,4]$. В настоящей работе на основе предложенных ранее простых моделей [9-11] мы приведем альтернативные аналитические оценки $C_{Q}$ для однолистного графена (SLG), графеновой наноленты с зигзагообразными краями (ZNR) и одномерной цепочки атомов углерода (карбина).

Вычисление квантовой емкости объекта начинается с определения плотности заряда $Q\left(V^{*}\right)=e(p-n)$, наведенной на этом объекте (здесь $V^{*}=e V_{e l}-$ сдвиг электронных состояний в электростатическом поле, $V_{e l}$ - внешний электростатический потенциал, $e-$ величина заряда электрона, $p$ и $n-$ концентрации дырок и электронов). Затем в соответствии со стандартным определением полагают $C_{Q}=\partial Q\left(V^{*}\right) / \partial V_{e l}$. Как показано в [2], емкость $C_{Q}$ двумерной наноструктуры определяется формулой

$$
\begin{gathered}
C_{Q}=\left(e^{2} / 4 T S\right) \int_{0}^{\infty} \rho(\omega) A\left(\omega, V^{*}\right) d \omega, \\
A\left(\omega, V^{*}\right)=\operatorname{sch}^{2}\left[\left(\omega-V^{*}\right) / 2 T\right]+\operatorname{sch}^{2}\left[\left(\omega+V^{*}\right) / 2 T\right],
\end{gathered}
$$

где $\omega$ - энергетическая переменная, $\rho(\omega)$ - плотность состояний, нормированная на один атом (здесь и далее при $V^{*}=0$ химический потенциал совпадает с точкой Дирака, принимаемой за начало отсчета энергии, так что $\rho(\omega)=\rho(-\omega)), T-$ температура в энергетических единицах, $S$ - площадь, приходящаяся на один поверхностный атом. Интересно отметить следующее. Во-первых, $C_{Q}^{0} \equiv C_{Q}\left(V^{*}=0\right) \neq 0, Q\left(V^{*}=0\right)=0$. Во-вторых, $\left(\partial C_{Q} / \partial V^{*}\right)_{V^{*}=0}=0$ и $\left(\partial^{2} C_{Q} / \partial V^{* 2}\right)_{V^{*}=0}<0$. Поэтому значение $C_{Q}^{0}$ следует рассматривать как минимальную (затравочную) квантовую емкость при $V^{*} \rightarrow 0$. И наконец, если верхняя граница сплошного спектра структуры равна $\omega_{\max }$, то при $V^{*} \gg \omega_{\max } \gg T$ получаем $C_{Q} \sim e^{2} / 2 S T \operatorname{ch}^{2}\left(V^{*} / 2 T\right) \rightarrow 0$, где мы учли, что для графеновых структур (точнее, для р-орбитали) имеем $\int_{0}^{\omega_{\max }} \rho(\omega) d \omega=1$

Начнем с бесконечного листа графена, для которого в [9] предложена следующая модель плотности состояний: $\rho_{\mathrm{SLG}}(\omega)=c|\omega| / t^{2}$ при $|\omega| \leqslant t, \rho_{\mathrm{SLG}}(\omega)=c|\omega|$ при $t<|\omega| \leqslant 3 t$ и $\rho_{\mathrm{SLG}}(\omega)=0$ при $|\omega|>3 t$, где $t-$ энергия перехода электрона между ближайшими соседями, $c=2 /(1+2 \ln 3)-$ нормировочный множитель, определяемый исходя из условия $\int_{0}^{3 t} \rho_{\mathrm{SLG}}(\omega) d \omega=1$. Подставляя $\rho_{\mathrm{SLG}}(\omega)$ в $(1)$, получим

$$
\begin{gathered}
C_{Q}^{\mathrm{SLG}}=C_{Q 1}^{\mathrm{SLG}}+C_{Q 2}^{\mathrm{SLG}}, \quad C_{Q 1,2}^{\mathrm{SLG}}=\left(e^{2} / 4 T S\right) I_{1,2}\left(\tau, v^{*}\right), \\
I_{1}\left(\tau, v^{*}\right)=\left(c / t^{2}\right) \int_{0}^{\tau} x A\left(x, v^{*}\right) d x \\
I_{2}\left(\tau, v^{*}\right)=c \int_{\tau}^{3 \tau} A\left(x, v^{*}\right) \frac{d x}{x}
\end{gathered}
$$

где $x=\omega / 2 T, \tau=t / 2 T, v^{*}=V^{*} / 2 T$. Интеграл $I_{1}\left(\tau, v^{*}\right)$ вычисляется точно:

$$
\begin{gathered}
I_{1}\left(\tau, v^{*}\right)=c \tau^{-2} B\left(\tau, v^{*}\right) \\
B\left(\tau, v^{*}\right)=\left(\tau\left[\operatorname{th}\left(\tau-v^{*}\right)+\operatorname{th}\left(\tau+v^{*}\right)\right]\right. \\
\left.-\ln \frac{\operatorname{ch}\left(\tau-v^{*}\right) \operatorname{ch}\left(\tau+v^{*}\right)}{\operatorname{ch}^{2} v^{*}}\right)
\end{gathered}
$$


Перейдем теперь к оценке интеграла $I_{2}\left(\tau, v^{*}\right)$. Полагая $\quad I_{2}\left(\tau, v^{*}\right)=I_{2}^{(-)}\left(\tau, v^{*}\right)+I_{2}^{(+)}\left(\tau, v^{*}\right), \quad$ где $I_{2}^{( \pm)}=\int^{3 t} d x / x \operatorname{ch}^{2}\left(x \pm v^{*}\right)$, и интегрируя по частям, получим ряды

$$
\begin{aligned}
& I_{2}^{( \pm)}\left(\tau, v^{*}\right)=c\left(\frac{\operatorname{th}\left(3 \tau \pm v^{*}\right)}{3 \tau}-\frac{\operatorname{th}\left(\tau \pm v^{*}\right)}{\tau}\right) \\
& \mp c \sum_{n=2}^{\infty}[(n-1) !]\left(\frac{\ln \operatorname{ch}\left(3 \tau \pm v^{*}\right)}{(3 \tau)^{n}}-\frac{\ln \operatorname{ch}\left(\tau \pm v^{*}\right)}{\tau^{n}}\right) .
\end{aligned}
$$

Полагая $T \sim 300 \mathrm{~K}, t \sim 3 \mathrm{eV}$ и сохраняя первые два члена в $I_{2}^{( \pm)}\left(\tau, v^{*}\right)$, получаем $I_{2}(\tau, 0) \approx-0.04 c$, так что $\left|I_{2}(\tau, 0)\right| \ll I_{1}(\tau, 0) \approx 0.28 c$. В пределе $\tau \gg v^{*}$ имеем $I_{2}\left(\tau, v^{*}\right) \sim 8 c \operatorname{ch}\left(2 v^{*}\right) I(\tau)$,

$I(\tau)=\int_{\tau}^{3 \tau}\left(e^{-2 x} / x\right) d x=\operatorname{Ei}(6 \tau)-\operatorname{Ei}(3 \tau) \approx \exp (-2 \tau) / 2 \tau$,

где $\operatorname{Ei}(. .$.$) - интегральная показательная функция [12].$ Тогда

$$
I_{2}\left(\tau, v^{*}\right) \approx 4 c \tau^{-1} \operatorname{ch}\left(2 v^{*}\right) \exp (-2 \tau) \ll I_{1}\left(\tau, v^{*}\right) \approx 2 c / \tau .
$$

Пренебрегая вкладом $I_{2}\left(\tau, v^{*}\right)$, запишем

$$
C_{Q}^{\mathrm{SLG}} \approx \frac{4 c e^{2} T}{3 \sqrt{3} a^{2} t^{2}} B\left(t, V^{*}\right)
$$

где $B\left(t, V^{*}\right)$ дается выражением (3) и учтено, что $S=3 \sqrt{3} a^{2} / 4, \quad a=1.42 \AA-$ расстояние между ближайшими соседями в графене. Отсюда при комнатной температуре получаем $C_{Q}^{0} \approx 1.6 \mu \mathrm{F} / \mathrm{cm}^{2}$.

Сравним (4) с выражением для квантовой емкости $\bar{C}_{Q}$, полученным в $[3,6]$. Учитывая, что скорость Ферми $v_{\mathrm{F}}=3 a t / 2 \hbar$, где $\hbar-$ приведенная постоянная Планка, в обозначениях настоящей работы имеем

$$
\begin{gathered}
\bar{C}_{Q}=\left(8 e^{2} T / 9 \pi a^{2} t^{2}\right) F\left(V^{*}, T\right), \\
F\left(V^{*}, T\right)=\ln \left[2\left(1+\operatorname{ch}\left(V^{*} / T\right)\right)\right] .
\end{gathered}
$$

В пределе $\tau \gg v^{*}$ имеем $B\left(\tau, v^{*}\right) \approx \ln 2\left(1+\operatorname{ch}\left(2 v^{*}\right)\right)$, откуда

$$
C_{Q}^{\mathrm{SLG}} / \bar{C}_{Q}=\pi \sqrt{3} /(1+2 \ln 3) \approx 1.7 .
$$

Подчеркнем, однако, что при выводе выражения для $\bar{C}_{Q}$ в $[3,6]$ допущена известная непоследовательность. Действительно, с одной стороны, низкоэнергетическое приближение распространяется на всю область интегрирования от 0 до $\infty$ (см. (1)), что равносильно переходу к пределу $t \rightarrow \infty$, так как плотность состояний графена занимает интервал $(-3 t, 3 t)$. С другой стороны, величина $v_{\mathrm{F}}=3 a t / 2 \hbar$ считается постоянной.

При $v^{*} \sim \tau$ и $v^{*} \geqslant \tau$ ситуация меняется кардинальным образом (см. рисунок). При комнатной температуре $F\left(V^{*}, T\right)=V^{*} / T$, а $B\left(V^{*}, T\right) \propto \exp \left(-\left(V^{*}-t\right) / T\right)$

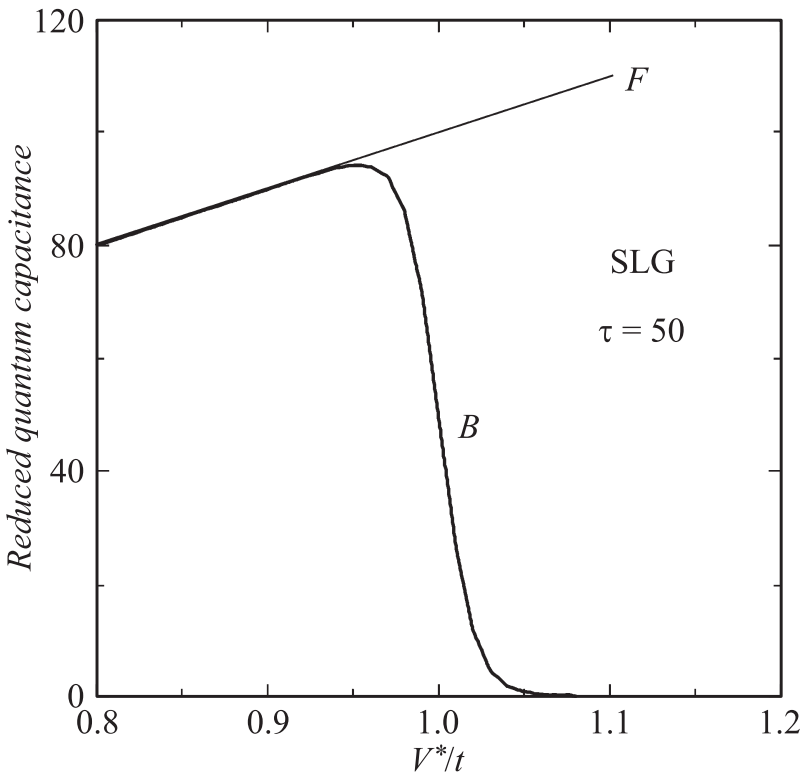

Зависимость функций $B\left(V^{*}, T\right)$ (жирная линия) и $F\left(V^{*}, T\right)$ (тонкая линия) для однолистного графена (SLG) от отношения $V^{*} / t$ при $\tau=t / 2 T=50$. Изображен только ограниченный участок графика, демонстрирующий расхождение между $B\left(V^{*}, T\right)$ и $F\left(V^{*}, T\right)$. При $V^{*} / T \leqslant 90\left(V^{*} / t \leqslant 0.9\right)$ имеем $B\left(V^{*}, T\right)=F\left(V^{*}, T\right)$.

(последняя зависимость уже обсуждалась в замечаниях к формуле (1)).

Перейдем к оценкам квантовой емкости $C_{Q}^{\mathrm{ZNR}}$ графеновой 2D-наноленты с зигзагообразными краями, электронный спектр которой не имеет щели и занимает энергетический интервал $\left(-\omega_{\max }, \omega_{\max }\right)$, где $\omega_{\max }=t(\sqrt{17}+1) / 2[10]$. ZNR содержит два сорта атомов углерода (см. рис. 1 работы [10]): внешние атомы, принадлежащие кромкам наноленты и имеющие двух ближайших соседей, плотность состояний которых будем обозначать как $\rho_{0}(\omega)$, и внутренние атомы, имеющие трех ближайших соседей, плотность состояний которых равна $\rho_{1}(\omega)$. Тогда плотность состояний элементарной ячейки $\rho_{\mathrm{ZNR}}(\omega)=\rho_{0}(\omega)+\rho_{1}(\omega)$, где значения $\rho_{0}(\omega)$ и $\rho_{1}(\omega)$ приведены в [10]. Из рис. $3, a$ работы [10] следует, что при $|\omega / t| \ll 1$ выполняется неравенство $\rho_{1}(\omega) \gg \rho_{0}(\omega)$. Тогда $\rho_{\mathrm{ZNR}}(\omega) \approx 1 / 4 \pi t T \sqrt{x \tau}(x \ll \tau)$ и

$$
C_{Q}^{\mathrm{ZNR}} \approx \frac{e^{2}}{8 \pi S t \sqrt{\tau}} J\left(v^{*}\right), \quad J\left(v^{*}\right)=\int_{0}^{\tau} A\left(x, v^{*}\right) \frac{d x}{\sqrt{x}},
$$

где учтено, что площадь элементарной ячейки ZNR равна $2 S$ и значения $\rho_{0}(\omega)$ и $\rho_{1}(\omega)$ в $(5)$ в 2 раза больше, чем в [10], так как здесь мы нормируем плотность состояний на два спиновых состояния. Тогда $A\left(x, v^{*}\right) \sim 8 \operatorname{ch}\left(2 v^{*}\right) \exp (-2 x)$. При $\tau \gg 1, v^{*} \ll \tau$ находим $J\left(v^{*}\right) \sim 8 \sqrt{\pi / 2} \operatorname{ch}\left(2 v^{*}\right)$ [11], откуда получаем

$$
C_{Q}^{\mathrm{ZNR}} \sim \frac{4 e^{2} \operatorname{ch}\left(V^{*} / T\right)}{3 \sqrt{6 \pi} a^{2} t} \sqrt{T / t} .
$$


При $v^{*} \gg \tau$ получаем $J\left(v^{*}\right) \sim 4 \sqrt{\tau} / \operatorname{ch}^{2}\left(v^{*}\right)$ и

$$
C_{Q}^{\mathrm{ZNR}} \sim \frac{2 e^{2}}{3 \pi \sqrt{3} a^{2} t \operatorname{ch}^{2}\left(V^{*} / 2 T\right)} .
$$

Таким образом, при $v^{*} \sim \tau$ рост $C_{Q}^{\mathrm{ZNR}}$ с увеличением $V^{*}$ сменяется спадом.

Отметим, что сопоставление выражений (6) и (7) с полученными в [3,4] результатами затруднительно: в [3] нанолента рассматривалась как одномерная структура, в [4] квантовая емкость равна $C_{Q}^{\prime}=e^{2} D\left(V^{*}\right)$, однако выражения для плотности состояний $D$ не приводится.

Обратимся теперь к одномерным карбинам [11] и рассмотрим сначала простейший случай кумулена атомной цепочки с двойными связями атомов углерода $(\cdots=\mathrm{C}=\mathrm{C}=\ldots)$ и плотностью состояний (на один атом) вида $\rho_{\text {сит }}(\omega)=2 / \pi \sqrt{4 t^{2}-\omega^{2}}$ при $\omega^{2} \leqslant 4 t^{2}$ и $\rho_{\text {сит }}(\omega)=0$ при $\omega^{2}>4 t^{2}$. Тогда

$$
C_{Q}^{c u m}=\left(e^{2} / 2 \pi T a\right) \bar{J}\left(v^{*}\right), \quad \bar{J}\left(v^{*}\right)=\int_{0}^{2 \tau} \rho(x) A\left(x, v^{*}\right) d x,
$$

где для одномерной структуры величина $C_{Q}^{c u m}$ нормируется на единицу длины, т.е. на постоянную цепочки $a$. Полагая при $\left|2 \tau-v^{*}\right| \gg 1$ в качестве грубой оценки $\bar{J}\left(v^{*}\right) \sim 8 \operatorname{ch}\left(2 v^{*}\right) \exp (-4 \tau)$, получим

$$
C_{Q}^{c u m} \sim\left(2 e^{2} / \pi T a\right) \exp \left(-\left|2 t-V^{*}\right|\right) .
$$

Вновь имеем кроссовер при $V^{*} \sim 2 t$.

Воспользовавшись результатами работы [11], можно показать, что для полиина (цепочки углеродных атомов с чередующимися тройными и одинарными связями $(\ldots \equiv \mathrm{C}-\mathrm{C} \equiv \mathrm{C}-\mathrm{C} \equiv \ldots))$ квантовая емкость $C_{Q}^{p o l} \approx C_{Q}^{c u m}$.

Итак, нами получены оценки квантовых емкостей $C_{Q}\left(V^{*}, T\right)$ для SLG, ZNR и карбинов. Для проверки справедливости исходных моделей и полученных в их рамках результатов необходимы дополнительные экспериментальные исследования. В заключение отметим, что в недавно появившихся работах [13-15] вопрос о квантовой емкости графеновых структур обсуждается в связи с проблемой суперконденсаторов и хранения энергии.

\section{Финансирование работы}

Один из авторов (ПВБ) признателен финансовой поддержке Министерства науки и высшего образования РФ в ходе реализации проекта „Фундаментальные основы механики, систем контроля и управления беспилотных авиационных систем с формообразующими конструкциями, глубоко интегрированными с силовыми установками, и уникальными свойствами, не применяемыми сегодня в пилотируемой авиации“ (№ FEFM-2020-0001).

\section{Конфликт интересов}

Авторы заявляют, что у них нет конфликта интересов.

\section{Список литературы}

[1] Luryi S. // Appl. Phys. Lett. 1988. V. 96. N 6. P. 501-503.

[2] John D.L., Castro L.C., Pulfrey D.L. // J. Appl. Phys. 2004. V. 96. N 9. P. $5180-5164$.

[3] Fang T., Konar A., Xing H., Jena D. // Appl. Phys. Lett. 2007. V. 91. N 9. P. 092109.

[4] Guo J., Yoon Y., Ouyang Y. // Nano Lett. 2007. V. 7. N 7. P. 1935-1940.

[5] Kliros G.S. // Rom. J. Inform. Sci. Technol. 2010. V. 13. N 3. P. 332-341.

[6] Cheremisin M.V. // Physica E. 2015. V. 69. N 1. P. 153-158.

[7] Alisultanov Z.Z., Reis M.S. // EPL. 2016. V. 113. N 2. P. 28004.

[8] Trabelsi A.B.G., Kusmartsev F.V., Forrester D.M., Kusmartseva O.E., Gaifullin M.B., Cropper P., Oueslati M. // J. Mater. Chem. C. 2016. V. 4. N 24. P. 5829-5838.

[9] Давыдов С.Ю., Сабирова Г.И. // ФТТ. 2011. Т. 53. В. 3. С. 608-616.

[10] Давыдов С.Ю., Зубов А.В. // ФТП. 2020. Т. 54. В. 2. С. $166-$ 170.

[11] Давыдов С.Ю. // ФТП. 2019. Т. 53. В. 7. С. 971-977.

[12] Градштейн И.С., Рызик И.М. Таблицы интегралов, сумм, рядов и произведений. М.: Наука, 1971. 1108 с.

[13] Su F., Huo L., Kong Q., Xie L., Chen C. // Catalysts. 2018. V. 8. N 10. P. 444-457.

[14] Xu Q., Yang G., Fan X., Zheng W. // ASC Omega. 2019. V. 4. N 8. P. 13209-13217.

[15] Sruthi T., Tarafder K. // Bull. Mater. Sci. 2019. V. 42. P. 257. https://doi.org/10.1007/s12034-019-1952-8 